\title{
Cognitive-behavioural therapy $v$. mirtazapine for chronic fatigue and neurasthenia: randomised placebo-controlled trial
}

\author{
Bjarte Stubhaug, Stein Atle Lie, Holger Ursin and Hege R. Eriksen
}

\section{Background}

Single interventions in chronic fatigue syndrome have shown only limited effectiveness, with few studies of comprehensive treatment programmes.

\section{Aims \\ To examine the effect of a comprehensive cognitive- behavioural treatment (CCBT) programme compared with placebo-controlled mirtazapine medication in patients with chronic fatigue, and to study the effect of combined medication and CCBT.}

\section{Method}

A three-armed randomised clinical trial of mirtazapine, placebo and a CCBT programme was conducted to investigate treatment effect in a patient group $(n=72)$ with chronic fatigue referred to a specialist clinic. The CCBT programme was compared with mirtazapine or placebo therapy for 12 weeks, followed by 12 weeks treatment with a mixed crossover-combination design. Assessments were done at 12 weeks and 24 weeks.

\section{Results}

By 12 weeks the treatment effect was significantly better in the group initially receiving CCBT, as assessed with the Fatigue Scale $(P=0.014)$ and the Clinical Global Impression Scale $(P=0.001)$. By 24 weeks the treatment group initially receiving CCBT for 12 weeks followed by mirtazapine for 12 weeks showed significant improvement compared with the other treatment groups on the Fatigue Scale $(P<0.001)$ and the Clinical Global Impression Scale $(P=0.002)$. Secondary outcome measures showed overall improvement with no significant difference between treatment groups.

\section{Conclusions}

Multimodal interventions may have positive treatment effects in chronic fatigue syndrome. Sequence of interventions seem to be of importance.

\section{Declaration of interest}

The trial received an unrestricted grant from Organon AS, who provided the placebo-controlled medication.
Neurasthenia as a medical term and diagnosis has been known since the 1870s, describing the ill-explained conditions of fatigue, fatiguability, muscular weakness and mental hypersensitivity. ${ }^{1}$ Since 1980 the labels 'chronic fatigue syndrome' and 'myalgic encephalopathy' have dominated the international debate and the research in this field, with several different case definitions. ${ }^{2-4}$ Systematic reviews of all interventions for treatment and management of chronic fatigue syndrome demonstrate mixed results in effectiveness. $^{5,6}$ Behavioural interventions, graded exercise and cognitive-behavioural therapy appear to reduce symptoms and improve function. Most studies report only minor systematic effects of single-treatment regimens in chronic fatigue.

We wanted to examine the possible effects of a comprehensive cognitive-behavioural treatment (CCBT) programme compared with medication, and also the effect of this programme combined with medication. We had found sleep disturbance and depressive symptoms to be prevalent comorbid symptoms in neurasthenia, as also reported in chronic fatigue. ${ }^{8}$ Mirtazapine was chosen as the trial medication because of its positive effect on normal sleep ${ }^{9,10}$ and positive effect on depressive symptoms. ${ }^{1-13}$ We have not found any previous study of mirtazapine in neurasthenia or chronic fatigue syndrome, but for fibromyalgia positive effects have been reported for pain, sleep complaints and depression. ${ }^{14}$

\section{Method}

The clinical study population consisted of 72 patients with chronic fatigue complaints, fulfilling ICD-10 research criteria for neurasthenia (code F48.0). ${ }^{15}$ Illness definition was operationalised by examining ICD-10 criteria. These diagnostic criteria are the presence of persistent and distressing feelings of exhaustion after minor mental effort or of fatigue after minor physical effort, accompanied by one or more of the following symptoms: muscular aches or pains; dizziness; tension headache; sleep disturbance; inability to relax; and irritability. There should be an inability to recover through rest, relaxation or enjoyment; the duration should exceed 3 months, and there should be no organic mental disorder, affective disorder, or panic or generalised anxiety disorder. The included patients satisfied these ICD-10 criteria, allowing for mild depressive or anxiety symptoms clinically evaluated to be independent of or secondary to fatigue symptoms. The criteria for chronic fatigue syndrome, specified by the Centers for Disease Control (CDC), ${ }^{4}$ and the Oxford group $^{3}$ were also examined in this population: 65 of the 72 patients fulfilled case definition by the Oxford (British) criteria and 29 patients fulfilled the CDC (US) criteria.

The trial participants were recruited during 2001 from a specialist clinic in the catchment area of the study hospital (population 100000 , mixed suburban and rural) situated on the west coast of Norway, partly by referral from general practitioners to the psychosomatic out-patient clinic for evaluation and treatment of fatigue complaints $(n=44)$, and partly by interviews at the specialist clinic $(n=28)$ after positive screening of fatigue by questionnaire in the waiting rooms of general practitioners in the area. The two different subgroups (referred patients $v$. screened patients) did not differ on most of the demographic or outcome variables. $^{7}$

Patients went through structured interviews and clinical evaluations, including somatic and laboratory investigations. The interviews and assessments were done using the Schedules for Clinical $(\mathrm{SCAN})^{16}$ structured interview schedules, checklists 
for neurasthenia and chronic fatigue syndrome case definitions, in addition to general medical evaluation. Patients fulfilling inclusion criteria were consecutively assigned ascending numbers and randomised into two different treatment groups (CCBT or medication) by concealed randomisation at an external research site, by means of randomisation tables. The further randomisation of medication into mirtazapine and placebo was done by assigning the patients consecutively to mirtazapine/placebo medication by concealed randomisation in a double-blind trial.

\section{Instruments}

Fatigue and clinical global impression of severity were the primary outcome measurements in this trial; depression, quality of life, general health, illness perceptions and cardiorespiratory fitness were secondary measures.

\section{Fatigue}

The Fatigue Scale ${ }^{17}$ is a self-rating scale developed to measure the severity of fatigue. The scale has been found to be both reliable and valid.

\section{General health status}

General health was assessed by subjective health complaints, measured by the Subjective Health Complaint inventory (SHC). ${ }^{18}$ This consists of 29 items on subjective somatic and psychological complaints experienced during the past 30 days. This questionnaire has been tested and has satisfactory validity and reliability. ${ }^{18}$ Severity was scored on a four-point scale, from 0 (no complaints) to 3 (severe complaints).

\section{Quality of life}

Health-related quality of life was measured using the generic health status measure, the 36-item Short Form Health Survey $(\mathrm{SF}-36)^{19}$ for health situations during the past 4 weeks; the Norwegian version of the instrument was used. ${ }^{20}$ The SF-36 is a generic quality of life scale consisting of 36 items describing eight dimensions, which are aggregated to one physical and one mental health component. ${ }^{21}$

\section{Clinical Global Impression}

The Clinical Global Impression (CGI) ${ }^{22}$ dimensions of severity of illness (CGI-S) and improvement (CGI-I) were used, rated on a seven-point scale. Severity of illness was rated within the past week; global improvement was rated since admission to the study. Severity was assessed by clinical assessment, whereas CGI-I scores were based on patients' self-reports. The CGI-S and CGI-I correlation was analysed.

\section{Depression}

Symptoms of depression were rated using the 21-item Hamilton Rating Scale for Depression (HRSD). ${ }^{23,24}$

\section{IIIness perception}

The Revised Illness Perceptions Questionnaire (IPQ-R) ${ }^{25}$ was used to measure patients' illness perceptions. This questionnaire has demonstrated good reliability and validity across several illness groups and includes eight cognitive dimensions.

\section{Cardiorespiratory fitness}

Cardiorespiratory fitness was assessed by the Åstrand-Ryhming test (indirect test of maximal oxygen uptake $\left.\left(\mathrm{VO}_{2} \max \right)\right){ }^{26}$ performed on an ergometer bicycle.

\section{Safety assessments}

Safety assessments included a full clinical examination prior to inclusion in the trial, and spontaneously reported adverse events and measurements of vital signs and weight at each scheduled efficacy evaluation, in addition to laboratory tests. Adverse events were coded in the Medical Dictionary for Regulatory Activities (MedDRA) system, ${ }^{27}$ using the preferred term as the unit of registration.

\section{Timing of assessments}

Assessments were done at baseline, 12 weeks and 24 weeks (end of treatment). Assessments at 12 weeks, after completion of the CCBT programme or medication/placebo course, registered treatment effects in three groups: CCBT $v$. mirtazapine medication $v$. placebo medication. Assessments at 24 weeks included four groups: 12 weeks CCBT followed by 12 weeks mirtazapine; 12 weeks CCBT followed by 12 weeks placebo; mirtazapine (24 weeks) combined with $0+12$ weeks CCBT; placebo medication (24 weeks) combined with $0+12$ weeks CCBT. Assessments were done mainly by self-report questionnaires and measures.

\section{Interventions}

The interventions, administered by a team comprising a psychiatrist, physiotherapists and a psychiatric nurse, were as follows:

(a) A comprehensive cognitive-behavioural treatment programme for 12 weeks, consisting of two $1.5 \mathrm{~h}$ sessions of group therapy per week (cognitive-behavioural therapy $1.5 \mathrm{~h}$, body awareness therapy $1.5 \mathrm{~h}$ ), combined with a selfmanaged exercise programme with individually set daily exercise goals (minimal to mild/moderate), using an exercise diary for planned and executed activity.

(b) Medication (double-blind) for 12 weeks: mirtazapine (individually adjusted dosage to adverse events; standard dose 15$45 \mathrm{mg}$, allowing lower dose) $v$. inactive placebo (medication was dispensed for 2-week periods from the hospital pharmacy; tablets taken by the patients were registered and the returned tablets counted by the pharmacist).

After the initial 12 weeks, treatment was continued in a combined crossover and treatment-combination design. Patients on medication (mirtazapine or placebo) received the CCBT programme for the next 12 weeks; patients having completed the CCBT programme received medication (mirtazapine or placebo) for the following 12 weeks.

The cognitive-behavioural therapy group sessions (12 sessions of $1.5 \mathrm{~h}$ ) were conducted by a team of a trained psychiatrist and a psychiatric nurse, using a semi-structured therapy manual with assigned topics for each session, including weekly reports of exercise tasks. Body awareness therapy emphasises increased awareness of bodily sensations, breathing, posture, balance and muscular tension, and focuses on how increased body awareness can help gain greater control over physical and mental tension. ${ }^{28}$ Treatment was conducted by physiotherapists trained in this therapy.

\section{Statistical methods}

The sample size needed was based on statistical calculation of presumed treatment effect of one standard deviation (4.0/33) from mean value of Fatigue Scale score, ${ }^{17}$ level of significance 0.05 , power 0.80 ; the s.d. being calculated from a pilot population of 20 patients with neurasthenia (mean value 23.6/33, variance 16.24 , s.d. $=4.03)$. The target sample size for a three- and fourarmed study was calculated to be $64(16 \times 4)$. The actual sample size $(n=72)$ exceeded the minimum number. To model the 
structure of the therapy in the study, we designed the covariates to account for changes in the status of the individuals. Thus, at baseline all individuals had the same status, whereas at 12 weeks there were three categories (mirtazapine, placebo and CCBT) and at 24 weeks there were four categories (mirtazapine 24 weeks + CCBT 12 weeks; placebo 24 weeks + CCBT 12 weeks; CCBT 12 weeks + mirtazapine 12 weeks; and CCBT 12 weeks + placebo 12 weeks). To account for the repeated measures for the participants, individuals were entered in the model as a random variable (with an unstructured covariance structure). Other variables were entered as fixed effects. Outcome analyses were done using PROC MIXED in SAS version 8.0 for Windows. Other analyses were done in SPSS version 13.0. Data analysis was completed on an intention-to-treat basis, with last observation carried forward (LOCF) as end value in cases of protocol violation or premature discontinuation. We also analysed the data omitting the missing observations, and found virtually the same results as when using LOCF; these results are therefore not presented.

\section{Ethics}

The study was approved by the Regional Ethics Committee, the Norwegian Social Science Data Services (NSD) and the Norwegian Medicines Agency. The trial was registered with NSD prior to any patient inclusion. All participants were given written information about the trial and gave formal consent about participation. Prospectively defined stopping rules were medical conditions warranting treatment, medication conflicting with study protocol, and serious adverse events due to or suspected to be caused by medication.

\section{Masking (blinding)}

Medication was provided in the form of capsules of $15 \mathrm{mg}$ mirtazapine or inactive substance. The capsules were identical in appearance, with no smell or taste. Allocation to active medication or placebo was concealed by hiding the allocation code in a locked envelope at the clinical site (in case of an emergency requiring breaking of the code), to be returned unopened to the manufacturer/provider by the end of the study. No emergency need occurred. The code was broken by the medication provider at the end of the follow-up period, after 1-year follow-up investigations, questionnaire completion and data collection. Masking seemed successful among participants by reports of effect and adverse events in both groups. There was no evidence by clinicians or assessors of systematic impression of non-concealment. All data were entered into the research database prior to breaking the allocation code. The trial statistician executed the analysis using anonymous data.

\section{Results}

Patients were recruited and included consecutively over a period of 12 months. The treatment programme (24 weeks) was

\begin{tabular}{|c|c|c|c|c|c|}
\hline & $\begin{array}{l}\text { Mirtazapine }(24 \text { weeks) } \\
+ \text { CCBT (12 weeks) } \\
\text { (n=25) }\end{array}$ & $\begin{array}{c}\text { Placebo ( } 24 \text { weeks) } \\
+ \text { CCBT ( } 12 \text { weeks) } \\
\text { (n=24) }\end{array}$ & $\begin{array}{c}\text { CCBT (12 weeks) } \\
+ \text { Mirtazapine (12 weeks) } \\
(n=11)\end{array}$ & $\begin{array}{c}\text { CCBT (12 weeks) } \\
+ \text { Placebo (12 weeks) } \\
(n=12)\end{array}$ & $\begin{array}{l}\text { Total } \\
(n=72)\end{array}$ \\
\hline \multicolumn{6}{|l|}{ Gender } \\
\hline Male & 6 & 3 & 2 & 2 & 13 \\
\hline Female & 19 & 21 & 9 & 10 & 59 \\
\hline Fatigue scale score: mean (s.d.) & $24.76(4.53)$ & $25.54(3.87)$ & $24.91(5.96)$ & $24.33(4.75)$ & $24.97(4.53)$ \\
\hline CGI score: mean (s.d.) & $4.84(0.80)$ & $4.92(0.72)$ & $5.18(0.60)$ & $5.27(0.79)$ & $4.99(0.75)$ \\
\hline SHC total score: mean (s.d.) & $23.56(9.29)$ & $26.02(10.55)$ & $27.36(13.00)$ & $27.00(10.10)$ & $25.54(10.35)$ \\
\hline Age, years: mean (s.d.) & $44.92(8.41)$ & $44.96(8.46)$ & $47.18(10.64)$ & $51.17(7.26)$ & $46.32(8.75)$ \\
\hline \multicolumn{6}{|l|}{ SF-36 score: mean (s.d.) } \\
\hline Physical & $26.39(9.70)$ & $30.65(12.16)$ & $31.13(10.54)$ & $27.74(12.92)$ & $28.94(11.31)$ \\
\hline Mental & 43.37 (11.31) & $34.32(11.98)$ & $37.19(13.05)$ & $44.93(14.91)$ & $39.37(13.06)$ \\
\hline HRSD score: mean (s.d.) & $13.32(4.79)$ & $15.92(3.66)$ & $14.00(2.00)$ & $14.64(3.04)$ & $14.51(3.92)$ \\
\hline CRF, $\mathrm{O}_{2} \mathrm{ml} / \mathrm{kg}$ : mean (s.d.) & $27.14(7.16)$ & $26.18(5.30)$ & $24.78(6.05)$ & $22.62(6.44)$ & $25.77(6.35)$ \\
\hline
\end{tabular}

Table 2 Difference between the therapies: results from a mixed model

\begin{tabular}{|c|c|c|c|c|c|c|c|c|c|c|c|c|c|}
\hline & \multirow[b]{2}{*}{$n$} & \multicolumn{3}{|c|}{ CGI score } & \multicolumn{3}{|c|}{ Fatigue Scale score } & \multicolumn{3}{|c|}{ HRSD score } & \multicolumn{3}{|c|}{$\mathrm{CRF}, \mathrm{O}_{2} \mathrm{ml} / \mathrm{kg}$} \\
\hline & & Mean & $(95 \% \mathrm{Cl})$ & $P$ & Mean & $(95 \% \mathrm{Cl})$ & $P$ & Mean & $(95 \% \mathrm{Cl})$ & $P$ & Mean & $(95 \% \mathrm{Cl})$ & $P$ \\
\hline 12 weeks & & & & $0.005^{a}$ & & & $0.047^{\mathrm{a}}$ & & & $0.64^{\mathrm{a}}$ & & & $0.25^{\mathrm{a}}$ \\
\hline Mirtazapine & 25 & 4.0 & (3.7-4.3) & 0.046 & 22.7 & (21.4-24.1) & 0.34 & 12.6 & (11.4-13.8) & 0.36 & 26.6 & (23.5-29.7) & 0.29 \\
\hline CCBT & 23 & 3.7 & $(3.1-4.2)$ & 0.001 & 21.0 & $(18.1-24.0)$ & 0.014 & 12.9 & $(10.1-15.7)$ & 0.54 & 24.6 & $(17.4-31.9)$ & 0.10 \\
\hline Placebo & 24 & 4.4 & $(3.9-4.9)$ & Ref. & 23.7 & $(21.0-26.5)$ & Ref. & 13.5 & $(10.9-16.1)$ & Ref. & 29.5 & $(22.6-36.4)$ & Ref. \\
\hline 24 weeks & & & & $0.001^{a}$ & & & $0.004^{a}$ & & & $0.075^{a}$ & & & $0.75^{a}$ \\
\hline Mirtazapine + CCBT & 25 & 4.6 & $(4.3-4.8)$ & 0.40 & 23.7 & (22.4-25.0) & 0.66 & 12.7 & $(11.6-13.9)$ & 0.13 & 27.6 & $(24.8-30.4)$ & 0.64 \\
\hline CCBT + placebo & 12 & 3.8 & $(3.2-4.4)$ & 0.045 & 23.3 & (20.1-26.5) & 0.55 & 14.0 & $(10.8-17.1)$ & 0.74 & 25.0 & (17.0-33.0) & 0.31 \\
\hline CCBT + mirtazapine & 11 & 3.4 & $(2.8-4.0)$ & 0.002 & 18.7 & $(15.4-22.0)$ & $<0.001$ & 10.8 & $(7.7-13.9)$ & 0.018 & 26.0 & $(17.7-34.2)$ & 0.44 \\
\hline Placebo + CCBT & 24 & 4.4 & $(3.9-4.9)$ & Ref. & 24.2 & $(21.4-27.1)$ & Ref. & 14.4 & (11.8-17.1) & Ref. & 29.0 & $(22.0-36.0)$ & Ref. \\
\hline
\end{tabular}


conducted through two periods, with a 6-month interval. Fortythree patients took part in the first programme and 29 in the second (total $n=72$ ). No significant difference was observed between treatment groups on baseline values of outcome measures (Table 1).

A total of 119 patients were eligible for the trial; 32 were excluded because of somatic or psychological disorders, and 15 people were unwilling to use medication as required by the study protocol. Baseline and treatment measurement data were available from 72 participants (Fig. 1).

There were significant improvements at 12 weeks in the CCBT group compared with the mirtazapine and placebo groups in terms of Fatigue Scale $(P=0.014)$ and CGI $(P=0.001)$ ratings (Table 2). End-of-treatment data at 24 weeks showed statistically significant improvement in the treatment group where the initial 12 weeks of CCBT was followed by 12 weeks of mirtazapine, compared with the reference group, which received placebo for 24 weeks and CCBT for the last 12 weeks. The improvement was significant for scores on the Fatigue Scale $(P<0.001)$, CGI $(P=0.002)$ and HRSD $(P=0.018)$, and was accompanied by a significant reduction in scores on the SHC, pseudoneurology sub-scale $(P=0.011)$ (Table 3$)$. Measures of health-related quality of life, using the SF-36 (two-component scale), showed significant improvement on the SF-36 mental sub-scale by the end of treatment (24 weeks) in the treatment group taking mirtazapine for 24 weeks plus CCBT for 12 weeks $(P=0.045)$ (Table 4$)$.

Data showed no consistent overall change in illness perception in the study population from baseline to end of study (24 weeks). A paired $t$-test showed significant changes in the illness perception dimensions of cyclical timeline $(P<0.001)$, illness consequences $(P=0.002)$ and illness coherence $(P=0.002)$, irrespective of treatment group. The one-way analysis of variance (ANOVA; SPSS version 13.0) showed a difference between groups on the illness perception of cyclical timeline $(P=0.028)$, but the post hoc comparisons (Sheffé) showed no difference between specific treatment groups.
Subjective global improvement of fatigue complaints was registered by self-report questionnaire of changes during treatment, CGI-I (7-points scale) and was correlated with the CGI-S assessment by clinician. Correlation analysis $(n=60)$ showed a significant correlation: $r=0.68(P<0.0001)$. Self-reported CGI-I scores showed an overall increase (mean difference 0.70, $P<0.001$ ), indicating improvement across the treatment groups, but with no significant difference in improvement between groups when analysed by one-way ANOVA or post hoc comparison.

Cardiorespiratory fitness, assessed by indirect measurement of maximal oxygen uptake, showed a tendency towards overall improvement in all groups following the total treatment programme ( $P=0.08$ at 12 weeks, $P=0.07$ at 24 weeks, compared with baseline values), in an unadjusted model with time effect only.

All patients taking mirtazapine and $45 \%$ of patients taking placebo reported at least one adverse event, irrespective of dosage or length of adverse event. The four most frequently reported adverse events in the mirtazapine group were sedation (56\%), increased appetite $(31 \%)$, weight increase $(33 \%)$ and restless legs syndrome $(19 \%)$. In the placebo-treated group, the most frequently reported adverse events were headache $(17 \%)$, sedation $(11 \%)$, weight increase $(11 \%)$ and insomnia $(11 \%)$. The drugassociated adverse events (i.e. events occurring in at least 5\% of patients and at a rate at least twice that of the placebo group) were sedation, increased appetite, weight increase, restless legs syndrome and constipation (Fig. 2).

There was no serious change in vital signs (except weight increase, reported as an adverse event) or in laboratory parameters (including endocrine and electrolyte measures) in the total study population, and no systematic difference between study groups or between patients with active medication and patients on placebo treatment.

Patients violating protocol (by stopping taking medication or using adjunctive medication beyond protocol) were allowed to continue in the treatment programme, but data for analysis of outcome and treatment effect were systematically based on LOCF

\begin{tabular}{|c|c|c|c|c|c|c|c|c|c|c|c|c|c|}
\hline & \multirow[b]{3}{*}{$n$} & \multicolumn{12}{|c|}{ SHC score } \\
\hline & & \multicolumn{3}{|c|}{ Total } & \multicolumn{3}{|c|}{ Musculoskeletal } & \multicolumn{3}{|c|}{ Pseudoneurology } & \multicolumn{3}{|c|}{ Gastrointestinal } \\
\hline & & Mean & $(95 \% \mathrm{Cl})$ & $P$ & Mean & $(95 \% \mathrm{Cl})$ & $P$ & Mean & $(95 \% \mathrm{Cl})$ & $P$ & Mean & $(95 \% \mathrm{Cl})$ & $P$ \\
\hline 24 weeks & & & & $0.58^{a}$ & & & $0.38^{\mathrm{a}}$ & & & $0.043^{a *}$ & & & $0.37^{a}$ \\
\hline Mirtazapine + CCBT & 25 & 24.1 & $(21.7-26.6)$ & 0.99 & 9.9 & $(8.8-11.1)$ & 0.37 & 7.2 & $(6.4-8.0)$ & 0.031 & 4.5 & $(3.6-5.4)$ & 0.081 \\
\hline CCBT + placebo & 12 & 24.5 & $(19.4-29.6)$ & 0.88 & 10.2 & $(8.1-12.3)$ & 0.34 & 7.9 & $(6.3-9.5)$ & 0.34 & 3.8 & $(1.9-5.8)$ & 0.44 \\
\hline CCBT + mirtazapine & 11 & 20.6 & $(15.3-25.9)$ & 0.23 & 8.2 & $(6.0-10.3)$ & 0.38 & 6.4 & $(4.8-8.1)$ & $0.011^{*}$ & 3.6 & $(1.6-5.6)$ & 0.60 \\
\hline Placebo + CCBT & 24 & 24.1 & $(20.1-28.1)$ & Ref. & 9.2 & $(7.5-10.9)$ & Ref. & 8.7 & $(7.5-10.0)$ & Ref. & 3.0 & $(1.5-4.5)$ & Ref. \\
\hline
\end{tabular}

CCBT, comprehensive cognitive-behavioural treatment; Ref., reference category; SHC, Subjective Health Complaint inventory.

Table 4 Difference between the therapies as assessed by the 36-item Short Form Health Survey: results from a mixed model

\begin{tabular}{|c|c|c|c|c|c|c|c|}
\hline & \multirow[b]{2}{*}{$n$} & \multicolumn{3}{|c|}{ SF-36 Mental sub-scale } & \multicolumn{3}{|c|}{ SF-36 Physical sub-scale } \\
\hline & & Mean & $(95 \% \mathrm{Cl})$ & $P$ & Mean & $(95 \% \mathrm{Cl})$ & $P$ \\
\hline 24 weeks & & & & $0.19^{a}$ & & & $0.34^{\mathrm{a}}$ \\
\hline Mirtazapine + CCBT & 25 & 42.6 & $(39.1-46.0)$ & $0.045^{\star}$ & 28.4 & $(25.4-31.4)$ & 0.20 \\
\hline CCBT + placebo & 12 & 41.0 & (33.7-48.2) & 0.19 & 27.1 & $(21.6-32.5)$ & 0.12 \\
\hline CCBT + mirtazapine & 11 & 37.1 & $(29.4-44.9)$ & 0.76 & 31.0 & $(25.2-36.8)$ & 0.89 \\
\hline Placebo + CCBT & 24 & 35.9 & $(30.2-41.6)$ & Ref. & 31.4 & $(27.0-35.8)$ & Ref. \\
\hline
\end{tabular}




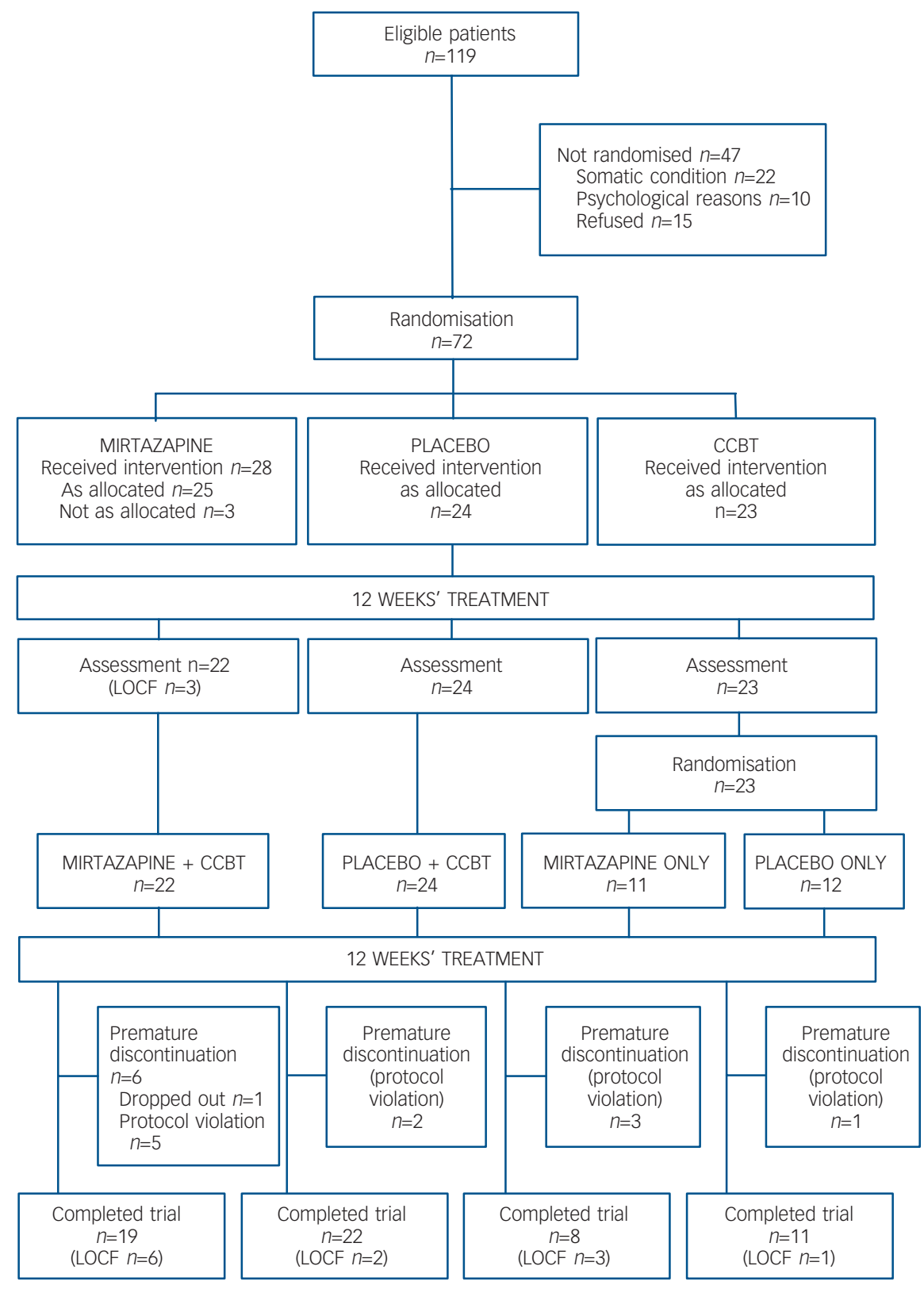

Fig. 1 Study profile. CCBT, comprehensive cognitive-behavioural treatment; LOCF, last observation carried forward.

data at the time of protocol violation. Other missing data from premature discontinuation were analysed by conservative model/ intention-to-treat analysis.

A source of bias and imprecision of the study findings was the outcome assessment (CGI-S) done by the psychiatrist (B.S.) responsible for treatment interventions. This was resolved by the use of self-report questionnaires on other outcome measurements, including self-reports of global improvement (CGI-I), which was correlated with the CGI-S measurements by the clinical assessor, showing significant correlation $(r=0.68, P<0.0001)$.

\section{Discussion}

This study has a complex design, and some of the findings can be questioned in view of both the complex combination of interventions and the small number in each treatment group. Power analysis of the results supports the significance of our positive findings, although type 2 errors cannot be ruled out for some of the negative findings owing to the low number of participants.

The findings from the study indicate that a comprehensive treatment programme based on multimodal interventions may have positive treatment effects in patients with chronic fatigue and neurasthenia, being superior to medication or placebo alone and being more effective as part of a combination treatment. The 12-week comprehensive treatment programme (cognitive group therapy, body awareness therapy and graded exercise) was significantly more effective than mirtazapine or placebo. The addition of mirtazapine (12 weeks) to the initial comprehensive treatment sequence showed a further treatment effect significantly superior $(P<0.001)$ to the other combinations of treatment, including the opposite sequence of medication first, followed by the comprehensive treatment programme. It seems that the timing of the different intervention sequences is of importance: our findings indicate that an initial non-pharmacological 'priming' by a cognitive-oriented treatment programme prior to medication 


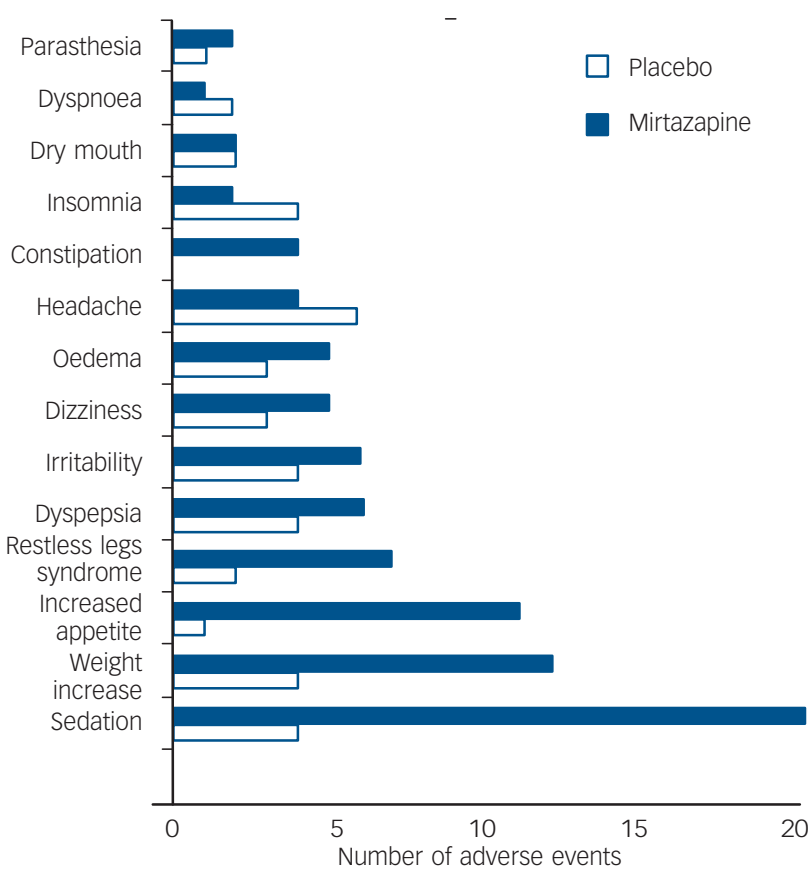

Fig. 2 Adverse events.

gives the best treatment effect. This is consistent with the results of a simulation analysis of the self-regulation of fatigue, in which the most effective treatment is stage-dependent with an initial period of delay before active intervention. ${ }^{29}$ It is possible that an introductory treatment phase with a cognitive-behavioural approach, focusing on an integrative model of fatigue, emphasising cognitive appraisal and body awareness, and the importance of regular, adjusted physical activity, brings better understanding of and better compliance with pharmacological treatment.

The findings are consistent for the primary outcome measures Fatigue Scale and CGI at 12 weeks and 24 weeks, and also for the HRSD by the end of the study at 24 weeks. Subjective Health Complaints showed a significant difference favouring the combination CCBT followed by mirtazapine on the pseudoneurological items scale, including tiredness (as would be expected, as fatigue symptoms showed reduction as measured by the Fatigue Scale). Quality of life outcome measures (SF-36) showed a slight but significant effect on mental health on the two-component aggregated scale, favouring the group using mirtazapine for 24 weeks. This effect on general mental health could be related to the active medication mirtazapine's positive effect on sleep difficulties reported by most patients, in addition to the antidepressant effect and general psychotropic action.

Illness perceptions were subject to change during the treatment programme. Many patients with fatigue complaints showed activity-avoidant behaviour based on misperceptions of their illness. These illness perceptions were the basis for our cognitive therapeutic approach aiming at modifying avoidance behaviour and excessive rest. Our data showed a reduction in illness perceptions on the dimensions of cyclical timeline, illness consequence and illness coherence, indicating a change in illness perceptions towards a more open-ended mental picture of the fatigue symptoms and neurasthenic illness, and a less rigid model of the condition, accepting it more as a complex disorder than as a disease with a single cause. These changes were found across the different treatment groups - as would be expected, since all groups received the cognitive treatment intervention.
Our study indicates that an effective treatment of chronic fatigue syndrome and neurasthenia should be based on a broad and comprehensive approach, using and combining treatment interventions already proved to be effective, with particular emphasis on the timing and sequence of the various interventions, in addition to the active participation and exercise by the patients themselves.

Bjarte Stubhaug, Haukeland University Hospital, Helse Bergen HF, Bergen; Stein Atle Lie, Holger Ursin, Research Centre for Health Promotion, Faculty of Psychology, University of Bergen, Bergen; Hege R. Eriksen, Department of Education and Health Promotion \& Research Centre for Health Promotion, University of Bergen, Bergen, Norway

Correspondence: Dr Bjarte Stubhaug Division of Psychiatry, Haukeland University Hospital, N-5021 Bergen, Norway. Email: bjarte.stubhaug@ helse-bergen.no

First received 9 Jan 2007, final revision 13 May 2007, accepted 19 Jul 2007

\section{References}

1 Beard G. Neurasthenia, or nervous exhaustion. Boston Med Surg J 1869; 3 217-21

2 Holmes GP, Kaplan JE, Gantz NM, Komaroff AL, Schonberger LB, Straus SE Jones SF, Dubois RE, Cunningham-Rundles C, Pahwa S. Chronic fatigue syndrome: a working case definition. Ann Intern Med 1988; 108: 387-9.

3 Sharpe MC, Archard LC, Banatvala JE, Borysiewicz LK, Clare AW, David A, Edwards RH, Hawton KE, Lambert HP, Lane RJ, McDonald EM, Mowbray JF, Pearson DJ, Peto TEA, Preedy VR, Smith AP, Smith DJ, Taylor DJ, Tyrrell DA, Wessely S, White PD. A report - chronic fatigue syndrome: guidelines for research. J R Soc Med 1991; 84: 118-21.

4 Fukuda K, Straus SE, Hickie I, Sharpe MC, Dobbins JG, Komaroff A. The chronic fatigue syndrome: a comprehensive approach to its definition and study. International Chronic Fatigue Syndrome Study Group. Ann Intern Med 1994; 121: 953-9.

5 Whiting $P$, Bagnall AM, Sowden AJ, Cornell JE, Mulrow CD, Ramirez G. Interventions for the treatment and management of chronic fatigue syndrome: a systematic review. JAMA 2001; 286: 1360-8.

6 Chambers D, Bagnall AM, Hempel S, Forbes C. Interventions for the treatment, management and rehabilitation of patients with chronic fatigue syndrome/myalgic encephalomyelitis: an updated systematic review. J $R$ Soc Med 2006; 99: 506-20.

7 Stubhaug B, Tveito TH, Eriksen HR, Ursin H. Neurasthenia, subjective health complaints and sensitization. Psychoneuroendocrinology 2005; 30: 1003-9.

8 Hickie I, Lloyd A, Wakefield D, Parker G. The psychiatric status of patients with the chronic fatigue syndrome. Br J Psychiatry 1990; 156: 534-40.

9 Winokur A, Sateia MJ, Hayes JB, Bayles-Dazet W, MacDonald MM, Gary KA Acute effects of mirtazapine on sleep continuity and sleep architecture in depressed patients: a pilot study. Biol Psychiatry 2000; 48: 75-8.

10 Aslan S, Isik E, Cosar B. The effects of mirtazapine on sleep: a placebo controlled, double-blind study in young healthy volunteers. Sleep 2002; 25 677-9.

11 Kasper S. Efficacy of antidepressants in the treatment of severe depression: the place of mirtazapine. J Clin Psychopharmacol 1997; 17 (suppl 1): S19-28.

12 Holm KJ, Markham A. Mirtazapine: a review of its use in major depression. Drugs 1999; 57: 607-31.

13 Freynhagen $\mathrm{R}$, Muth-Selbach $\mathrm{U}$, Lipfert $\mathrm{P}$, Stevens MF, Zacharowski $\mathrm{K}$ Tölle TR, von Giesen JH. The effect of mirtazapine in patients with chronic pain and concomitant depression. Curr Med Res Opin 2006; 22: 257-64.

14 Samborski W, Lezanska-Szpera M, Rybakowski JK. Open trial of mirtazapine in patients with fibromyalgia. Pharmacopsychiatry 2004; 37: 168-70.

15 World Health Organization. The ICD-10 Classification of Mental and Behavioural Disorders: Diagnostic Criteria for Research. WHO, 1993.

16 World Health Organization. Schedules for Clinical Assessment in Neuropsychiatry (SCAN). WHO, 1992.

17 Chalder T, Berelowitz G, Pawlikowska T, Watts L, Wessely S, Wright D, Wallace EP. Development of a fatigue scale. J Psychosom Res 1993; 37: 147-

18 Eriksen HR, Ihlebaek $\mathrm{C}$, Ursin H. A scoring system for subjective health complaints (SHC). Scand J Public Health 1999; 27: 63-72.

19 Ware JE, Snow KK, Kosinski M. SF-36 Health Survey Manual and Interpretation Guide. QualityMetric Inc, 2000 
20 Loge JH, Kaasa S, Hjermstad MJ, Kvien TK. Translation and performance of the Norwegian SF-36 Health Survey in patients with rheumatoid arthritis. I. Data quality, scaling assumptions, reliability, and construct validity. J Clin Epidemiol 1998; 51: 1069-76.

21 Ware JE, Kosinski M. SF-36 Physical and Mental Health Summary Scales: A Manual for Users of ver 1. QualityMetric Inc, 2001.

22 Guy W. CGI Clinical Global Impressions. In ECDEU Assessment Manual for Psychopharmacology (Revised) 217-22. National Institute for Mental Health 1976

23 Hamilton M. A rating scale for depression. J Neurol Neurosurg Psychiatry 1960; 23: 56-62.

24 Hamilton M. Development of a rating scale for primary depressive illness. $\mathrm{Br}$ Soc Clin Psychol 1967; 6: 278-96.
25 Moss-Morris R, Weinman J, Petrie KJ, Horne R, Cameron LD, Buick D. The Revised Illness Perception Questionnaire (IPQ-R). Psychol Health 2002; 17: $1-16$.

26 Astrand PO, Ryhming I. A nomogram for calculation of aerobic capacity (physical fitness) from pulse rate during sub-maximal work. J Appl Physiol 1954; 7: 218-21.

27 Brown EG, Wood L, Wood S. The medical dictionary for regulatory activities (MedDRA). Drug Saf 1999; 20: 109-17.

28 Skatteboe UB, Friis S, Hope MK, Vaglum P. Body awareness group therapy for patients with personality disorders. 1. Description of the therapeutic method. Psychother Psychosom 1989; 51: 11-17.

29 van Mens-Verhulst J, van Dijkum C, van Kuijk E, Lam N. The self-regulation of fatigue and associated complaints: an exploratory simulation. Patient Educ Couns 2003; 49: 53-7.

\section{psychiatry} in pictures
Lunacy and exercising (1818). By Friedrich Guimpel (1774-1839). Reproduced in Offentliche Rechenschaft by Ernst Horn (1778-1848)

Text by Dr Kai Sammet

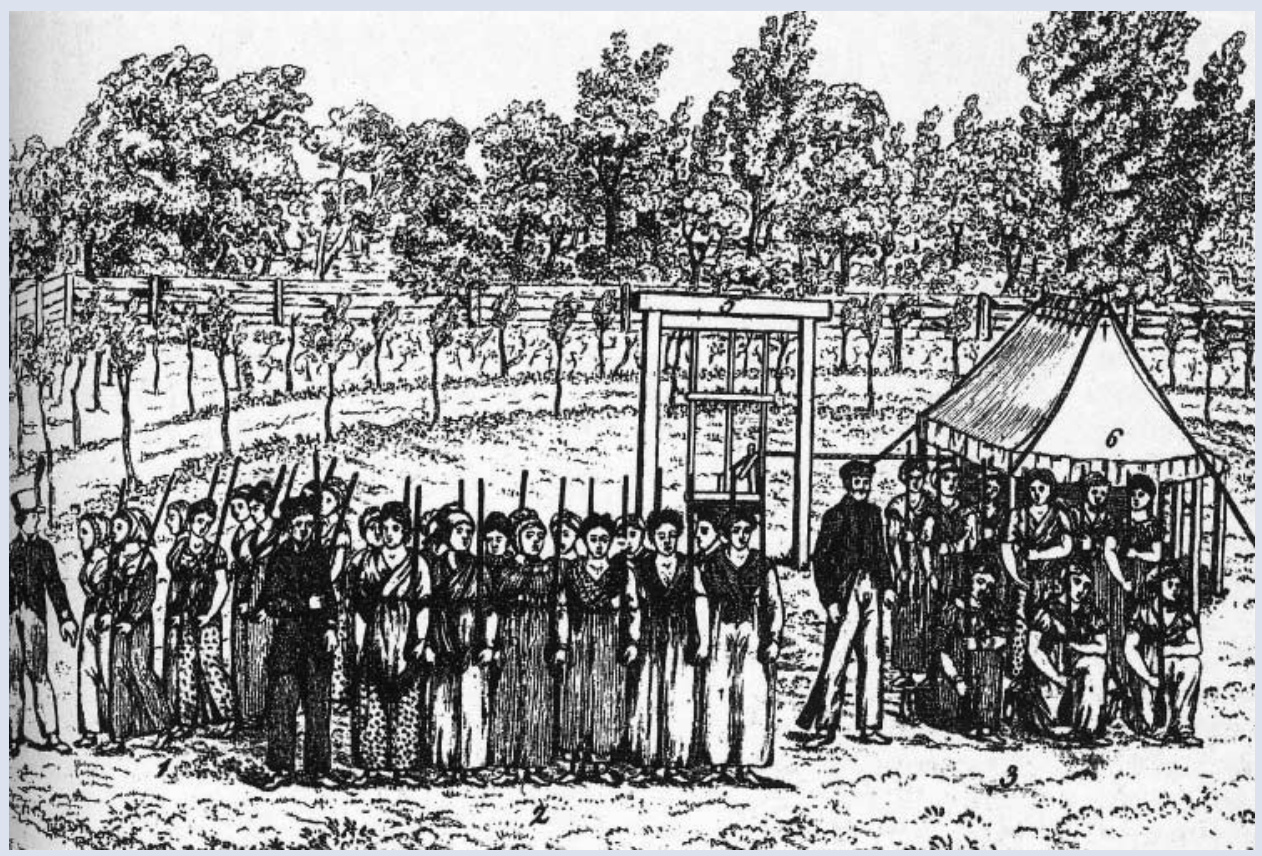

In his seminal Traité medico-philosophique sur I'aliénation mentale of 1801, the eminent French alienist, Philippe Pinel (1745-1826) held that 'the most secure and perhaps only guarantee to maintain sanity, good morals and order' in asylums should be 'mechanical work rigorously applied'. This image reflects Pinel's therapeutic philosophy. It shows female patients exercising with guns made of wood in the yard of the psychiatric ward of the Berlin Charité around 1810. The image appeared in a psychiatric treatise by Ernst Horn. He had studied in Jena and Gottingen, and was appointed professor at the Military Academy in Berlin in 1806. Horn was also given responsibility for the psychiatric ward in the Charite hospital, where he prescribed the type of therapeutic intervention illustrated in the picture. It was not a manifestation of Prussian militarism but stemmed from his theories on the origin of mental illness. Horn was convinced that orderliness and regularity helped to smooth emotions and to 'rectify' reason. Exercise was held to be especially important. Horn, along with J. B. Langermann and J. C. Reil, was one of the first psychiatrists in the German-speaking countries. 